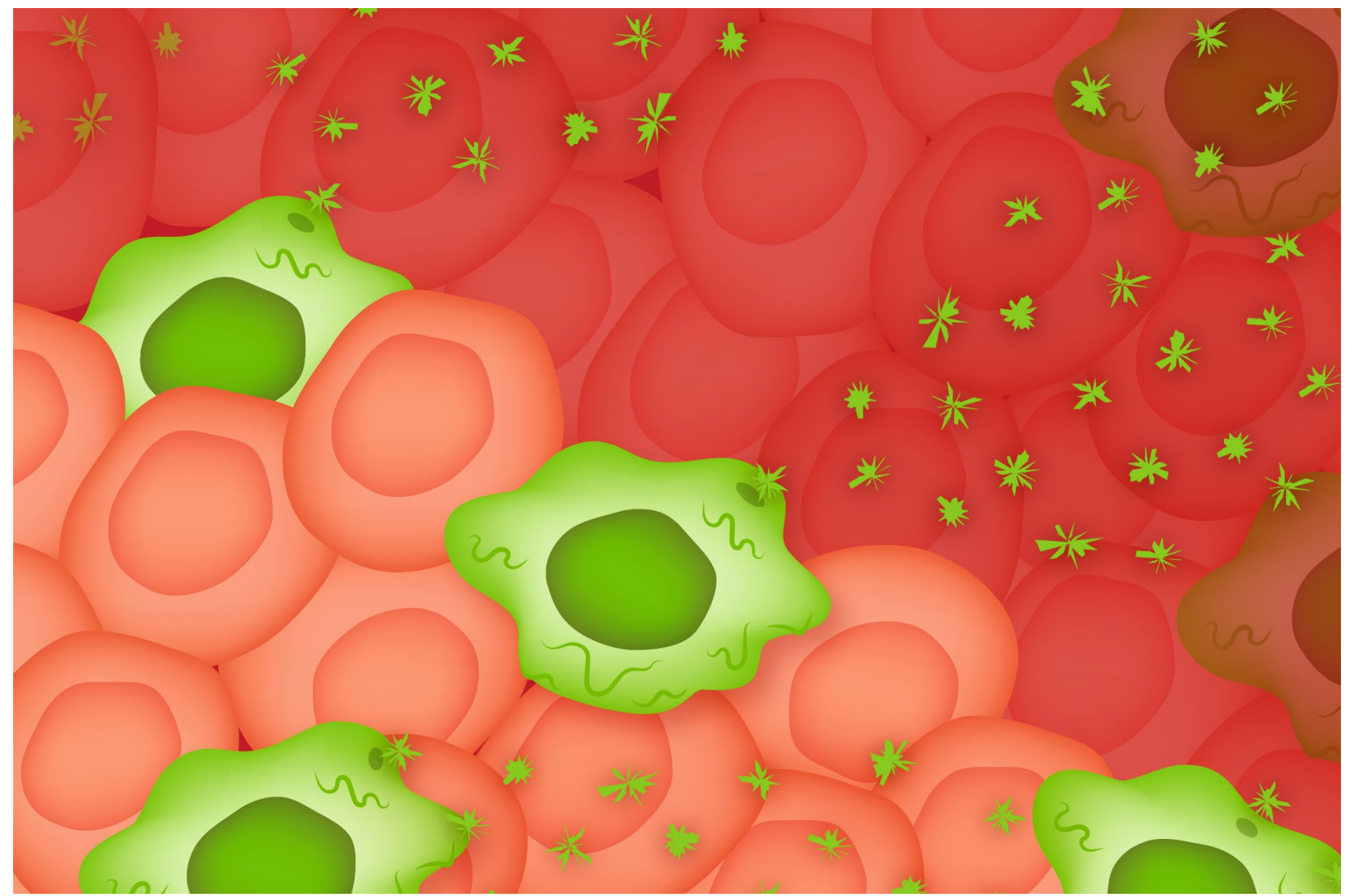

\title{
Send in the senolytics
}

Despite early failures in the clinic, the idea of anti-aging therapies that purge the body of dying cells is gaining traction with a raft of startups now focused on senescence.

\section{Elie Dolgin}

nity Biotechnology was one of the darlings of the nascent anti-aging biotech sector. With big-name investors, such as Jeff Bezos and Peter Thiel, an impressive lineup of academic founders and a market valuation that once reached $\$ 700$ million, the company was, in effect, the industry's standard-bearer for the therapeutic idea of destroying senescent cells to blunt the ravages of aging.

In August, that idea took a hit when Unity announced that its lead drug candidate had failed to beat a placebo in reducing joint pain and stiffness, according to interim results from a trial of patients with osteoarthritis of the knee. The company's stock plunged more than $60 \%$ on the news, nearly one-third of Unity staff were laid off, and its experimental drug UBX0101 - the first novel 'senolytic' agent ever to enter clinical testing - was swiftly abandoned.

That setback threw a pall over the entire senolytic field. A research note from Citi analyst Yigal Nochomovitz cited "substantially greater risk to the senolytic hypothesis." However, other experts were more sanguine about a class effect, highlighting issues with trial design, study population and UBX0101 itself - a small-molecule inhibitor of MDM2 (murine double minute 2) designed to boost the activity of the proapoptotic $\mathrm{p} 53$ protein - as possible explanations for study failure.

In the meantime, more than two dozen other startups (Table 1) continue to pursue approaches to target senescent cells - strategies that range from cell destruction and containment to senescence prevention and even reversal. "There are lots of different ways to tackle this, and I think that the initial failure of Unity's first clinical trial shouldn't be a discouragement," says Tim Cash, CSO of Senolytic Therapeutics. 


\section{Table 1 | Selected biotech companies focused on senolytics}

\begin{tabular}{|c|c|}
\hline Company (year founded) & Business focus/technology \\
\hline 1E Therapeutics (2020) & Antisense oligonucleotide-based senolytics \\
\hline Atropos Therapeutics (2018) & $\begin{array}{l}\text { Targeting transition between quiescence and senescence } \\
\text { (senescence after growth arrest, or SAGA) }\end{array}$ \\
\hline Cleara Biotech (2018) & Targeting FOXO4 to release proapoptotic $\mathrm{p} 53$ \\
\hline $\begin{array}{l}\text { Deciduous Therapeutics } \\
\text { (2018) }\end{array}$ & Activating immune cells to clear senescent cells \\
\hline Dialectic Therapeutics (2018) & $\begin{array}{l}\text { Systemic delivery of senolytic agents using proteolysis-targeting } \\
\text { chimeras (PROTACs) }\end{array}$ \\
\hline Dorian Therapeutics (2018) & Targeting USP16, a deubiquitination enzyme, to reverse senescence \\
\hline Eternans (2017) & FOXO4-binding peptide \\
\hline FoxBio (2018) & Targeting p53/FOXO4 prosurvival pathways in senescent cells \\
\hline Genome Protection (2018) & Stimulating innate immunity to eradicate genome-compromised cells \\
\hline Geras Bio (2020) & SASP inhibitors \\
\hline $\begin{array}{l}\text { Insilico Medicine/ } \\
\text { Taisho (2020) }\end{array}$ & Al target identification and generation/validation \\
\hline NRTK Biosciences (2020) & Synthetic optimization of approved drugs and supplements \\
\hline Numeric Biotech (2017) & Selective targeting of FOXO4-p53 \\
\hline Oisín Biotechnologies (2014) & Gene therapy with caspase- 9 activated in p16-positive cells \\
\hline Oncosence (2019) & $\begin{array}{l}\text { Monoclonal antibodies targeting tumor cells after inducing them to } \\
\text { senescence }\end{array}$ \\
\hline OneSkin (2016) & $\begin{array}{l}\text { Peptide that modulates senescence-related signaling pathways and } \\
\text { enhances DNA repair }\end{array}$ \\
\hline Recursion Pharma (2013) & Al drug discovery platform \\
\hline Rejuversen (2020) & $\begin{array}{l}\text { Antibody against PD-L2 that promotes immune-mediated clearance } \\
\text { of senescent cancer cells }\end{array}$ \\
\hline Rubedo Life Sciences (2018) & Small-molecule senolytics \\
\hline Senisca (2020) & Antisense oligonucleotides against splicing factors \\
\hline Senolytic Therapeutics (2017) & Senolytic and senomorphic drugs to treat fibrosis \\
\hline SIWA Therapeutics (2006) & Antibody against glycation surface molecule \\
\hline Unity Biotechnology (2011) & Targeting various senescence-related proteins ( $\mathrm{Bcl}-\mathrm{xL})$ \\
\hline
\end{tabular}

As Cash puts it, "the field is young, it's still early, and there's a lot left to be discovered and tried."

\section{Zombie cells}

The idea of therapeutically targeting senescence traces its roots back to the laboratory of Jan van Deursen, a molecular biologist who directed the mouse transgenic and knockout core facility at the Mayo Clinic for over 20 years.

In the early 2000s, van Deursen engineered a strain of mice with low levels of the mitotic checkpoint protein BubR1, which helps maintain the proper segregation of chromosomes during cell division. He expected the resulting chromosome instability to lead to tumor formation. But hardly any mice developed cancer. Instead, they just seemed to age prematurely ${ }^{1}$.

By the time the mice were a few months old, their eyes were pocked with cataracts, their skin was wearing thin, their muscles were wasting away, and - conspicuously - their organs were chock-full of senescent cells, trapped in a state of long-term cell-cycle arrest like mosquitoes in amber. The cells had stopped dividing, but refused to die. cellular existence - first described ${ }^{2}$ by biogerontologist Leonard Hayflick and cytogeneticist Paul Moorhead at the Wistar Institute in 1961 - was chiefly understood as a defense mechanism against cancer. In the face of replicative stress, cells were thought to enter senescence as a way to avoid becoming malignant.

But in van Deursen's mice, the cells seemed to have a dark side. They kept tumors at bay, but also fueled the aging process through a toxic brew of secreted interleukins, chemokines, growth factors, enzymes and other molecules that were
At the time, this zombie-like state of arousing local inflammation and damaging nearby cells. Judith Campisi, a cell biologist at the Buck Institute for Research on Aging in Novato, California who led one of the three teams that first described this complex network of immune-modulating molecules, gave the phenomenon a name: the senescence-associated secretory phenotype, or $\mathrm{SASP}^{3}$ (Fig. 1).

To many researchers, suppressing the SASP seemed like the most obvious way of combating the ills of senescence to treat cancer and diseases of aging. But in 2011, van Deursen - working with a Mayo colleague, gerontologist James Kirkland showed that one could simply do away with the senescent cells instead. Their paper detailed a transgenic strategy (devised by Kirkland and adapted from a technique ${ }^{4}$ pioneered by Philipp Scherer, a fat-cell researcher now at the University of Texas Southwestern Medical Center in Dallas) for selectively purging mice of cells in stable replicative arrest by targeting a protein characteristic of senescence: the tumor suppressor $\mathrm{p} 16^{\text {lnk4a }}\left(\right.$ ref. $\left.^{5}\right)$. In the rapidly aging mice, the senescent ouster retarded many aspects of the usual fast-tracked tissue degeneration.

Within weeks of that report, van Deursen had teamed up with serial biotech entrepreneur Ned David. They looped in Campisi, who was working on a similar transgenic mouse model for selective senescent cell destruction at the time, and Unity (initially known as Cenexys) was born. The van Deursen mice had lost their senescent cells through a trick of genetic engineering. Now the goal was to find drug compounds capable of doing the same thing in people.

Early leads mostly came from the world of oncology, where the aim is also to induce cell death. But whereas a cancer drug must eliminate every last tumor cell to avoid disease recurrence, a senolytic can still be effective if it purges only a meaningful fraction. And whereas the rapid pace of cancer development usually necessitates giving high doses of drug on a frequent basis, senescence targeting often works at lower doses administered intermittently. "It's a really hit-and-run approach," says Paul Robbins, a molecular biologist at the University of Minnesota Medical School in Minneapolis.

Unity's first promising candidate came from Campisi's lab, where researchers discovered that a small-molecule inhibitor of MDM2 called nutlin-3a had senolytic activity. (A form of that compound eventually became UBX0101.) Another lead emerged from a drug screen conducted by Daohong Zhou, a cancer researcher now at 


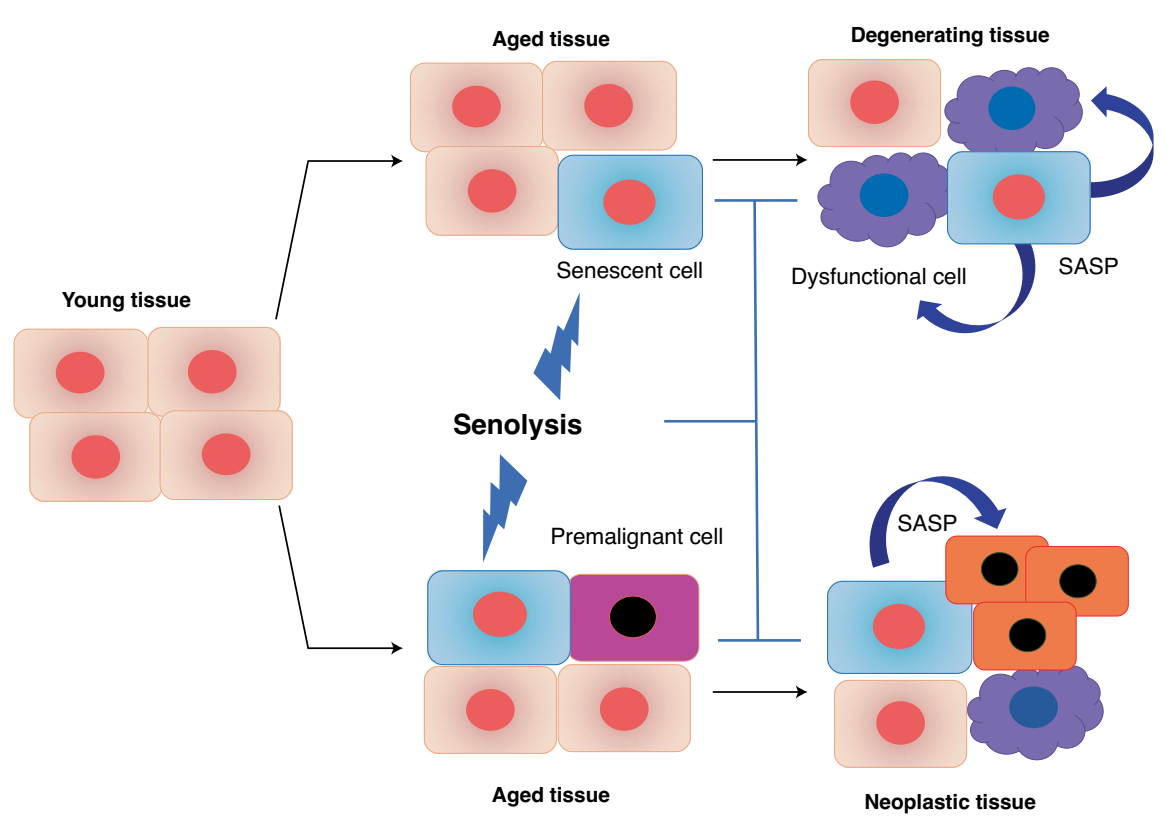

Fig. 1 | Targeting senescence. One strategy to interfere with aging is to prevent senescent cells from releasing SASP-associated factors that contribute to cancer and age-related disease.Credit: Judith Campisi, Buck Institute; adapted with permission.

the University of Florida at Gainesville, who in 2014 became a scientific cofounder of Unity. He and his colleagues had identified Abbott's navitoclax, an experimental inhibitor of the antiapoptotic B-cell lymphoma 2 (BCL-2) family of proteins, as a potent senolytic ${ }^{6}$. UBX1325, which targets one of these proteins, BCL-xL, entered phase 1 testing in October for the treatment of diabetic macular edema.

But Unity was hardly alone in its quest for senolytic agents. Kirkland soon embarked on a drug-repurposing effort, looking for approved agents with antisenescence activity; the most promising leads are now in clinical testing. And on the commercial front, many other companies soon formed, including one that has even managed to bring a senolytic product to market, albeit as a supplement not subject to the same regulations as medicinal drugs (Box 1).

Like Unity, most of these startups drew inspiration from van Deursen's 2011 paper $^{5}$ and a follow-up report ${ }^{7}$ showing the promise of senescent cell clearance to protect against age-related organ deterioration in normal aging mice as well. "I refer to those mice [in van Deursen's lab] as the mice that launched a thousand ships," says Matthew Scholz, cofounder and CEO of Oisín Biotechnologies, a senolytics-focused gene therapy company.

When it comes to raising funds, many of those senolytic 'ships' have hit rocky waters in recent months. Because of the UBX0101 trial failure, some investors - already burned once by the hype that surrounded the ill-fated anti-aging company Sirtris Pharmaceuticals (Nat. Biotechnol. 28, 185-186, 2010) and fearing a repeat with companies such as Unity - have shied away from pouring additional money into the antisenescence drug market.

Yet industry insiders say the problem with Unity's trial had more to do with the specifics of that drug and its study protocol than with the strategy of senolysis writ large. "Of course, we've been anxiously following their progress through the clinic, and we're disappointed too," says Lewis Gruber, CEO and CSO of SIWA Therapeutics, a company developing an antibody drug directed against a type of advanced glycation end product found on the surface of senescent cells. "But the actual technical results are, at least so far, not of concern to us," he says. "We don't see it as a problem for senolytics in general."

\section{Why Unity underdelivered}

For starters, MDM2 may not have been the best target for senescent cell destruction. The protein is one of the most important negative regulators of $\mathrm{p} 53$, responsible for the ubiquitination and degradation of the proapoptotic tumor suppressor. As such, inhibiting MDM2 risks unleashing indiscriminate cell killing in off-target, non-senescent tissues.

To avoid that kind of toxicity, Unity relied on a local delivery strategy. But according to biomedical engineer Jennifer Elisseeff from Johns Hopkins University in Baltimore, Maryland, local injections of UBX0101 are not sufficient to improve knee function in old mice with osteoarthritis. In her lab, systemic administration of another senolytic agent, navitoclax, was needed to tamp down the general inflammation in the body that was hampering tissue repair ${ }^{8}$.

Then there's the drug itself. Other senescence researchers say that, in their hands, MDM2 inhibitors such as nutlin-3a have only weak senolytic activity. And there's evidence from Campisi's lab to suggest that the drug could also work through attenuation of the SASP ${ }^{9}$. If that mechanism predominates over cell elimination, a single dose of UBX0101, as administered in the trial, might thus offer only temporary relief before the inflammatory secretome would come flaming back.

Unity executives, meanwhile, pin the blame on an unexpectedly large placebo response in the phase 2 study that may have obscured any treatment effect of UBX0101. But such a response was entirely predictable, experts say, given the known analgesic effects of saline injections in osteoarthritis ${ }^{10}$ and the short duration of the 12-week primary endpoint. Unity is expected to announce data from a 24-week follow-up period, as well as from another study that evaluated a two-dose regimen. Both analyses could conceivably demonstrate a therapeutic benefit, although, in light of the 12-week data, the company said that it does not plan to advance the program into pivotal studies.

Unity instead is now focusing its efforts on the development of its BCL-xL inhibitor UBX1325 for retinal diseases, with a preclinical research program ongoing for neurological conditions as well. And despite the early clinical stumble, Anirvan Ghosh, a former Biogen executive who joined Unity as CEO in March of this year, says he remains confident that the company can deliver. "The core biology is really strong," he says.

Many researchers are trying to improve on that biology, though. Zhou, for example, has focused on trying to make BCL-xL blockade safer for systemic administration. Working with his colleague Guangrong Zheng, a medicinal chemist at the University of Florida, Zhou took advantage of proteolysis-targeting chimeras (PROTACs) - heterobifunctional molecules consisting of a ligand for binding a protein of interest and a E3 ubiquitin ligase that tags the target for degradation - to convert navitoclax into a BCL-xL-directed agent that only induces the death of cells that express a particular ubiquitin ligase called cereblon.

Navitoclax is normally toxic to platelets, which rely on BCL-xL for survival. Yet 


\section{Box 1 | Skin in the game}

There is already one senescence-focused company that has brought a product to market, albeit not one subject to the same strict regulatory requirements as most therapeutic goods. On 21 October, OneSkin launched its first commercial product, a topical supplement that the company says can reduce the senescent cell burden in populations of skin cells by up to $40 \%$ in lab experiments.

CEO Carolina Reis de Oliveira and her colleagues discovered the supplement's active ingredient by phenotypically screening around 1,000 peptides, tracking markers of senescence in cultured skin cells. In parallel, they developed an algorithm that condenses DNA methylation patterns at some 2,200 sites across the genome of dermal fibroblast and keratinocyte cells into a single metric, a skin-specific predictor of biological age ${ }^{23}$.

Using this tool, the researchers led by CSO Alessandra Zonari, along with cofounders Mariana Boroni, a bioinformatician at the Brazilian National Cancer Institute in Rio de Janeiro, and Juliana Lott de Carvalho, a stem cell biologist at the Catholic University of Brasília - validated the ability of the screen's top lead to reverse skin biological age. In three-dimensional skin models and biopsy samples, the molecule - a

because platelets express cereblon only at low levels, the new PROTAC drug proved far more selective to senescent cells and thus tolerable in a mouse model ${ }^{11}$. "We have to have a better, safer, systemic therapy for senescent cell clearance," says Zhou, who together with Zheng cofounded a company called Dialectic Therapeutics to develop the strategy further.

\section{Potent peptides}

Others are taking a closer look at the idea of recruiting p53 in the fight against senescent cells. Yet, instead of targeting MDM2 as Unity's UBX0101 did, several startups are taking aim at forkhead box O4 (FOXO4), a transcription factor that normally sequesters p53 inside the nucleus, thereby neutralizing its apoptotic capacity.

In 2017, a team led by Peter de Keizer, a researcher specializing on aging at University Medical Center Utrecht in the Netherlands, described a cell-permeable peptide comprising part of FOXO4 that could disrupt this process ${ }^{12}$. By freeing p53 to exit the nucleus and engage apoptotic pathways in the cytoplasm of senescent short linear peptide dubbed OS-01 enhanced DNA repair mechanisms and downregulated senescence-related signaling pathways. Over time, skin cells treated with this peptide accumulated fewer markers of senescence, regardless of the inductive trigger.

An unpublished study in 22 older adults showed that, over the course of 12 weeks, the topical supplement improved skin barrier function by up to $18 \%$ and enhanced other aspects of skin health, such as elasticity, evenness and firmness. To Reis de Oliveira, the strengthening of the skin barrier suggests "a very good correlation between our in vitro data and what we see in people's actual skin."

Another trial is now planned in which participants will apply the OneSkin product for up to a year before donating a small sliver of skin behind the ear. Reis de Oliveira and her colleagues plan to conduct their methylomic analysis of skin aging to determine whether in fact the treatment leads to decreases in skin biological age in vivo.

At $\$ 120$ for a 50 -milliliter bottle - less with a subscription plan - OneSkin's product is not exactly delivering on the blockbuster revenue-generating promise of senescence-targeted therapeutics. But it's a start.

cells, the therapy, which incorporated right-handed amino acids for added potency, helped counteract frailty, hair loss and renal dysfunction in mice.

It was the first intentionally designed senolytic agent - and it attracted a lot of attention. One self-experimenter even started injecting himself with de Keizer's peptide and chronicling his experiences online. Along a more conventional drug development path, the Erasmus University Medical Center in Rotterdam, de Keizer's employer at the time, spun off a company called Numeric Biotech to commercialize the therapy. And Uğur Sezerman, a protein engineer at Acibadem University in Istanbul, Turkey, started his own company called Eternans centered around another peptide drug that directly binds to FOXO4, instead of to the FOXO4-interaction domain on p53. In preclinical experiments, both peptide strategies seem to accomplish the same therapeutic end result.

Working through his own startup, Cleara Biotech, de Keizer has also further optimized his peptide, introducing more unusual amino acids - some right-handed, some unnatural - to make the drug more stable and targeted to the post-transcriptionally modified form of $\mathrm{p} 53$ found in senescent cells. "We designed it specifically around the right type of p53," he says.

Before advancing that next-generation peptide into the clinic, however, de Keizer and his fellow Cleara cofounder, Marco Demaria of the University Medical Center Groningen in the Netherlands, are trying to better define which subtypes of senescent cells are worth targeting. Demaria previously identified various gene expression signatures linked to certain senescence-inducing stresses and cell types ${ }^{13}$. Others, including Campisi and her Buck colleagues, have characterized similar features using proteomic data ${ }^{14}$. But, says Demaria, "we are far from being able to define something as senescence-specific."

And therein lies one of the major problems for the field. To identify senescent cells, many researchers rely on imperfect proxies, such as $\beta$-galactosidase activity or increased expression of the cyclin-dependent kinase inhibitor p16. But none of these features is strictly limited to senescent cells, which raises the specter of on-target collateral damage. Plus, different subtypes invariably show different hallmarks of the senescence phenotype.

\section{Putting down a marker}

That means a single senolytic strategy is unlikely to work for all age-related conditions, says Nir Barzilai, an anti-aging researcher at Albert Einstein College of Medicine in New York. "We don't know enough about the commonalities in order to target with one drug all the senescent cells," he says. "We always talk about senescent cells as if every senescent cell is the same, but that's been a gross oversimplification."

As the search for biomarkers continues, some researchers are already looking into the possibility of developing diagnostic tests for determining whether a senescence-targeted therapy is working or not. At Eberhard Karls University in Tübingen, Germany, for example, a team led by oncologist Lars Zender and biomedical engineer Bernd Pichler has developed a radioactive form of $\beta$-galactosidase that can be tracked non-invasively in the body through positron emission tomography. A first-in-human trial is now evaluating the safety and imaging characteristics of the senescence-specific radiotracer in people with cancer.

Meanwhile, Campisi's team, led by former postdoc Christopher Wiley, recently discovered a biologically active signaling lipid that senescent cells synthesize as part of 
their inflammatory SASP. This lipid, a type of prostaglandin, is normally confined to the insides of senescent cells. But if a senolytic agent successfully bursts open its target cells, the lipid is liberated and becomes detectable in body fluids. The researchers have submitted a report showing that, in mice with drug-induced senescence, a dose of navitoclax caused levels of the prostaglandin to spike, both in blood and in urine ${ }^{15}$.

"We've got the first marker to know if your senolytic is working," says Wiley, a metabolism and senescence researcher now at the Jean Mayer USDA Human Nutrition Research Center on Aging at Tufts University in Boston. According to Wiley, at least two companies - a large pharmaceutical firm and a smaller diagnostics startup - have expressed interest in validating the lipid biomarker's predictive value in patients. Wiley is also probing vulnerabilities in senescence-associated lipid metabolism pathways, searching for targets amenable to direct cell-killing applications.

Elsewhere, several other outside-the-box strategies are being explored as senescencedirected lines of attack. At the Memorial Sloan Kettering Cancer Center (MSKCC) in New York, for example, cancer geneticist Scott Lowe and his colleagues have developed a senolytic cell therapy modeled after the kinds of chimeric antigen receptor (CAR)-T cells that have transformed cancer treatment.

Working with his colleague Michel Sadelain, a pioneer of T-cell engineering technologies, Lowe designed a CAR construct that targets urokinase-type plasminogen activator receptor (uPAR), a cell-surface protein that is broadly expressed by senescent cells. The researchers reported earlier this year that their uPAR-specific CAR-T cells could efficiently ablate senescent cells in mouse models of lung cancer and liver fibrosis ${ }^{16}$.

The mice did experience signs of general immune activation, consistent with other CAR-T cell approaches. And there was some on-target, off-senescence toxicity, owing to the expression of uPAR on some macrophages. But Lowe anticipates the therapy could be made safer by using lower doses of short-acting CAR-T cells. "You could imagine a scenario where your CAR-T cells come in, clear out the senescent cells, and then they disappear," he says.

Notably, that's the exact opposite of the strategy being pursued by most oncology-focused companies, which are now trying to develop more potent and durable CARs that are resistant to exhaustion (Nat. Biotechnol. 38, 3-5, 2020). "For a senolytic, non-cancer indication, because the cells aren't dividing, you may not need to have nearly as much potency and duration," Lowe says. Subpar CARs "might be exactly what you want."

Safety switches and combinatorial target-antigen recognition systems could further limit the opportunity for side effects, says Lowe, who is now exploring commercial opportunities for the technology. CAR-equipped natural killer (NK) cells, which tend to be shorter-lived and more amenable to off-the-shelf allogeneic administration, provide another option as well.

\section{A natural death}

Amit Sharma, an immunologist at the SENS Research Foundation, a longevity-focused non-profit organization in Mountain View, California, has other ideas for how to augment the innate antisenescence function of NK cells. "Imagine a way by which we can isolate somebody's NK cells, activate them in vitro, and then put them back into the body to remove the senescent burden," he says. Sharma has unpublished data showing that NK cells lose their ability to target senescent cells as they age, but can regain this function through treatment in a particular bath of interleukin molecules.

Then there is Oisín, whose gene therapy approach involves a DNA construct designed to kill senescent cells. The DNA plasmid encodes a suicide gene, caspase-9, whose activity is controlled by the promoter for $\mathrm{p} 16$, a gene involved in cell cycle regulation that is highly expressed in senescent cells. That genetic cargo is then encapsulated in a neutral-lipid nanoparticle that incorporates parts of the p14 fusion-associated small transmembrane (FAST) protein from a reptilian reovirus and the p15 FAST protein from a baboon virus. Thanks to this fusogenic polypeptide, the nanoparticle melds seamlessly with cells throughout the body - unloading its genetic payload directly into the cytoplasm, without any vesicular intermediaries. Once deposited, however, the DNA transgene only gets turned on in p16-positive cells. "It is targeting based on genetics," Scholz explains.

The gene alone doesn't trigger

cell death, though. For added safety,

a short course of drug therapy - in this case, a subtherapeutic dose of the immunosuppressant rapamycin - is needed to promote the dimerization of caspase-9, which then activates the lethal protein and clears away the senescent cells.

In unpublished work, Oisín scientists have found that their senolytic agent can reduce the number of p16-expressing kidney cells in the laboratory by about $50 \%$. According to Scholz, the company has teamed up with nephrologist Joseph Bonventre of Brigham and Women's Hospital in Boston to conduct in vivo studies in mouse models of acute kidney injury and chronic kidney disease, and Oisín is on track to launch trials for patients with various types of renal failure in the next two years. A spinoff company called OncoSenX is also developing a form of the same gene therapy platform for treating cancer, with the inducible caspase- 9 gene controlled by a $\mathrm{p} 53$ promoter.

A different kind of platform strategy comes from Senisca, a company spun off from Lorna Harries's lab at the University of Exeter Medical School in the United Kingdom. Harries built her academic career studying the ways in which splicing factors affect the aging process. And in recent years, her group has shown how modulation of splicing factor expression can rescue cells from a senescent state ${ }^{17}$. Now, through Senisca - which Harries founded earlier this year with her former $\mathrm{PhD}$ student Ben Lee - she is designing antisense oligonucleotides targeting certain splicing factors, or to the master control genes that regulate them, with the goal of resetting the alternative splicing patterns that contribute to cellular aging.

It's not just the antisense oligonucleotide modality that sets Senisca's approach apart, though. 1E Therapeutics, an Israeli startup, is also developing antisense-based senolytics - in their case, oligonucleotides that incorporate catalytically active nucleic acid sequences designed to enhance the inhibition of specific senescence-associated genes. But Harries's technology, she claims, actually seems to reverse senescence, not just slow the process or destroy the unwanted cells. In a lab dish at least, "we have a means of rejuvenating cells rather than just wiping them out," says Harries, who serves as CSO of the company.

Dorian Therapeutics, a 2018 spinout from Stanford University in California, similarly asserts that by blocking USP16, a deubiquitination enzyme involved both in inhibiting stem cell function and in promoting senescence, the company can retip the cellular balance toward self-renewal, both in aged tissues and in cell culture. "We really switch the entire genetic program of the cells into a more useful expression profile," says cofounder and CEO Maddalena Adorno, who first characterized USP16's role in the self-renewal and senescence pathways as a postdoc in Michael Clarke's cancer stem cell lab at Stanford in the early $2010 \mathrm{~s}^{18}$.

"It means that the older cells don't progress into aging as fast, so they work a bit better," she says, "and also that the stem cells become more active and regenerate more of the tissue." 


\section{Senescent slogans}

In a sector awash with buzzwords, Adorno and her cofounder, CSO Benedetta Nicolis di Robilant, have labeled their drugs "senoblockers." Other companies variously describe their treatments as senomorphics, senostatics, senomodulators and senosuppressors.

In the senomorphics game, for instance, is Geras Bio, a company cofounded by MSKCC's Lowe and Jesús Gil of Imperial College London. The startup is pursuing SASP inhibitors, the idea being to spare beneficial senescent cells while choking off the bad ones. As CEO Jordan Fridman explains, the SASP "is an interesting, complex secretome filled with a number of immune-modulating and microenvironment-shaping factors that can be friend or foe, really, depending on the setting." Senolytic Therapeutics, despite having 'lytic' embedded in its name, is also expanding its focus to target various molecular factors produced by senescent cells found in the fibrotic tissue of people with nonalcoholic fatty liver disease.

Then, claiming the mantle of senosuppression, is Atropos Therapeutics. The brainchild of MSKCC molecular biologist Andrew Koff, Atropos is developing small-molecule drugs that block a chromatin-remodeling enzyme called ATRX $(\alpha$-thalassemia mental retardation X-linked protein) from accumulating in nuclear foci, a critical step on the road to senescence.

According to Koff, the approach is intended to "slow the rate at which cells convert from quiescence into senescence," thereby giving the immune system a chance to clear senescent cells at a steady pace. Atropos partnered with Atomwise last year to find lead candidates using artificial intelligence-based drug discovery approaches. More recently, the company teamed up with the Estée Lauder Companies to screen natural product libraries as well.

Koff's lead indication under consideration is sarcopenia, a disease of muscle loss that affects at least $10 \%$ of the world's elderly population. But, like other companies in the space, Atropos is also exploring applications in oncology, the idea being to prevent the formation of senescent cancer cells so that other therapies can be more effective.

But what if senescence in cancer can be exploited? That's the hope of René Bernards, a cancer geneticist at the Netherlands Cancer Institute in Amsterdam, who has devised a strategy he calls the "one-two punch" approach to cancer therapy. By coaxing tumor cells into senescence with one treatment and then wiping out the cells with another, he hopes to solve the problem of therapy-induced senescence that is often responsible for cancer metastasis and disease relapse.

Bernards and his colleagues outlined the approach in a proof-of-concept study published last year ${ }^{19}$. Using a small-molecule inhibitor of the DNA-replication kinase CDC7, followed by treatment with sertraline, a blocker of the kinase mTOR (mammalian target of rapamycin), his team selectively induced senescence and then killed off liver cancer cells. Through a startup called Oncosence, Bernards and his cofounder, molecular oncologist Andrea Alimonti from ETH Zürich and the Università della Svizzera Italiana in Bellinzona, Switzerland, now plan to develop the strategy further.

So far, Oncosence has identified an antigen expressed across a range of different senescent cancer cell types - "and it's also independent of the initial senescence trigger," Bernards says. The company now plans to create a monoclonal antibody directed against this target.

Rejuversen, the latest startup from Manuel Serrano of the Institute for Research in Biomedicine in Barcelona, is similarly developing an antibody for senescent cancer cell clearance, but one designed to promote immune-mediated elimination rather than direct killing. The approach started on a hunch: just as tumor cells evade immune surveillance by expressing checkpoint molecules on their surface, Serrano explains, "we reasoned that perhaps senescent cells, when they survive for a long time in the body and become pathologic, they could also be protecting themselves with immunosuppressive ligands."

In collaboration with Susana Llanos from the Spanish National Cancer Research Center in Madrid, Serrano performed an unbiased screen of membrane-bound proteins and discovered that senescent cells are bedazzled in copies of programmed cell death ligand 2 (PD-L2), a lesser-known binding partner to the PD-1 checkpoint protein targeted by many of today's best-selling cancer immunotherapies. Rejuversen, which formed earlier this year around intellectual property licensed from Serrano's previous startup, Senolytic Therapeutics, now aims to commercialize a PD-L2-targeted antibody drug as a way to eliminate cancer cells that have become senescent following chemotherapy.

In a similar vein, Deciduous Therapeutics is also trying to expunge senescence via reactivation of endogenous immune surveillance mechanisms. But rather than target an antigen found on the surface of senescent cells, the company is focused on directly rousing the relevant immune cells instead. "We identified an immune population that, when stimulated, will remove senescent cells," says Anil Bhushan from the University of California, San Francisco, one of Deciduous's cofounders. Plus, he adds, "we can target it very specifically, without affecting other cell types." The company has designed therapeutic candidates directed toward this endogenous immune population and is now conducting preclinical testing in both ageand non-age-related disease models.

\section{Finding a repurpose}

Kirkland, for his part, opted for a quicker path to the clinic. After initially dabbling in the idea of developing senolytic fusion toxin proteins, and then struggling to conduct high-throughput drug screens for new molecules with anti-senescent-cell activity, he opted for a different course of action. Together with his Mayo colleagues Tamara Tchkonia and Yi Zhu, Kirkland looked for vulnerabilities in the signaling pathways that promote the survival of senescent cells. They performed RNA-interference screens and then evaluated approved drug compounds with known activity against those weak spots.

As Kirkland explains, "We purposely went for compounds that would hit multiple targets on the nodes" of the prosurvival signaling network. His team also focused on molecules with short half-lives "because we know that, in vitro at least, it takes ten days to six weeks for new senescent cells to form," he says, "and what we wanted were drugs that would act in a hit-and-run manner."

In 2015, the Mayo team, in collaboration with Robbins and University of Minnesota mouse geneticist Laura Niedernhofer, described one particularly potent senolytic drug combination: Sprycel (dasatinib), a leukemia drug from Bristol Myers Squibb that targets multiple kinases, plus the dietary supplement quercetin, a flavonoid concentrated in fruit skins. The researchers later identified fisetin ${ }^{20}$, another naturally occurring flavonoid, as an even better candidate than quercetin when used as a single-agent therapy.

Kirkland launched his first clinical trial in 2016, with dasatinib and quercetin or $\mathrm{D}+\mathrm{Q}$, as the combination has come to be known - given to survivors of hematopoietic stem cell transplants, a population prone to premature aging. The regimen proved safe, which "gave us some confidence to go ahead with more trials of the drugs," Kirkland says.

With colleagues at the Mayo Clinic and elsewhere, he then initiated D+Q studies in patients with age-related diseases of the kidneys, lungs and brain, as well as in otherwise healthy older women at risk for 
bone fractures. Initial results in patients with diabetic kidney disease ${ }^{21}$ and idiopathic lung fibrosis ${ }^{22}$ look promising - and fisetin trials are now ongoing as well. Two studies, for example, are evaluating the therapy's potential to alleviate complications of COVID-19.

But, as Robbins points out: "None of these compounds were selected initially for senolytic activity." And as such, they "most likely can be improved with medicinal chemistry." Through a new company called NRTK Biosciences - named after its founders, Niedernhofer and Robbins, plus its scientific advisors, Tchkonia and Kirkland - he has already created a more potent analog of fisetin, with plans to synthesize many more.

As with any new chemical entity, an improved version of fisetin will have to progress through the usual clinical development process. (Fisetin is available for purchase at health-food stores, but, as Kirkland points out, "We don't know what the downsides are going to be, and people should not be taking any of these agents outside of the context of a carefully controlled clinical trial.") Like UBX0101, the new analog could also fail for any number of reasons.

But the early success of D+Q at least shows that the concept of senolysis is possible is patients. And it gives anti-aging researchers hope that more pronounced benefits will be seen with therapeutics rationally designed to modulate senescence in some way. "It'll happen sooner or later," says Wiley. "The technology is just too good."

\section{Elie Dolgin}

Somerville, MA, USA.

Published online: 12 November 2020

https://doi.org/10.1038/s41587-020-00750-1
References

1. Baker, D. J. et al. Nat. Genet. 36, 744-749 (2004).

2. Hayflick, L. \& Moorhead, P. S. Exp. Cell Res. 25, 585-621 (1961)

3. Coppé, J.-P. et al. PLoS Biol. 6, e301 (2008).

4. Pajvani, U. B. et al. Nat. Med. 11, 797-803 (2005)

5. Baker, D. J. et al. Nature 479, 232-236 (2011)

6. Chang, J. et al. Nat. Med. 22, 78-83 (2016).

7. Baker, D. J. et al. Nature 530, 184-189 (2016).

8. Faust, H. J. et al. J. Clin. Invest. 130, 5493-5507 (2020).

9. Wiley, C. D. et al. Sci. Rep. 8, 2410 (2018).

10. Zhang, W., Robertson, J., Jones, A. C., Dieppe, P. A. \& Doherty, M. Ann. Rheum. Dis. 67, 1716-1723 (2008).

11. He, Y. et al. Nat. Commun. 11, 1996 (2020).

12. Baar, M. P. et al. Cell 169, 132-147.e16 (2017).

13. Hernandez-Segura, A. et al. Curr. Biol. 27, 2652-2660.e4 (2017).

14. Basisty, N. et al. PLoS Biol. 18, e3000599 (2020).

15. Wiley, C. D. et al. Preprint at Cell Metab. https://doi.org/10.2139/ ssrn.3528689 (2020).

$\square \quad$ 16. Amor, C. et al. Nature 583, 127-132 (2020).

17. Latorre, E., Ostler, E. L., Faragher, R. G. A. \& Harries, L. W. FASEB J. 33, 1086-1097 (2019).

18. Adorno, M. et al. Nature 501, 380-384 (2013). 19. Wang, C. et al. Nature 574, 268-272 (2019)

20. Zhu, Y. et al. Aging (Albany NY) 9, 955-963 (2017).

21. Hickson, L. J. et al. EBioMedicine 47, 446-456 (2019).

22. Justice, J. N. et al. EBioMedicine 40, 554-563 (2019).

23. Boroni, M. et al. Clin. Epigenetics 12, 105 (2020). 